\title{
Stability Analysis of a Nonlinear Difference Equation
}

\author{
Fatma Bozkurt \\ Department of Mathematics, Faculty of Education, Erciyes University, Kayseri, Turkey \\ Email: fbozkurt@erciyes.edu.tr
}

Received January 1, 2013; revised January 31, 2013; accepted February 9, 2013

\begin{abstract}
The local and global behavior of the positive solutions of the difference equation

$$
y_{n+1}=\frac{\alpha \cdot \mathrm{e}^{-y_{n}}+\beta \cdot \mathrm{e}^{-y_{n-1}}}{\gamma+\alpha \cdot y_{n}+\beta \cdot y_{n-1}}, n=0,1, \cdots
$$

was investigated, where the parameters $\alpha, \beta$ and $\gamma$ and the initial conditions are arbitrary positive numbers. Furthermore, the characterization of the stability was studied with a basin that depends on the conditions of the coefficients. The analysis about the semi-cycle of positive solutions has end the study of this work.
\end{abstract}

Keywords: Difference Equations; Stability Analysis; Semi-Cycle Solutions; Boundedness

\section{Introduction}

H. El-Metwally et al. [1] studied the global stability of the difference equation

$$
x_{n+1}=\alpha+\beta x_{n-1} \mathrm{e}^{-x_{n}}, n=0,1, \cdots,
$$

where the parameters $\alpha$ and $\beta$ are positive numbers and the initial conditions are arbitrary non-negative real numbers. This equation may be viewed as a model in Mathematical Biology, where $\alpha$ is the immigration rate and $\beta$ the population growth rate.

In [2] was investigated the globally asymptotically stability of the difference equation

$$
y_{n+1}=\frac{\alpha+\beta \mathrm{e}^{-y_{n}}}{\gamma+y_{n-1}}, n=0,1, \cdots,
$$

where the parameters $\alpha, \beta$ and $\gamma$ are positive numbers and the initial conditions are arbitrary non-negative numbers. In [3] the boundedness and the global asymptotic behavior of the difference equation

$$
y_{n+1}=\frac{\alpha \cdot \mathrm{e}^{-\left(n y_{n}+(n-k) y_{n-k}\right)}}{\beta+n y_{n}+(n-k) y_{n-k}}, n=0,1, \cdots,
$$

was shown, where $\alpha$ and $\beta$ are positive real numbers, $k \in\{1,2, \cdots\}$ and the initial conditions $y_{-k}, \cdots, y_{-1}, y_{0}$ are arbitrary numbers. Similar studies can be shown in [4-7].

The aim in this paper is to study the local and global behavior of the positive solutions of the difference equation

$$
y_{n+1}=\frac{\alpha \cdot \mathrm{e}^{-y_{n}}+\beta \cdot \mathrm{e}^{-y_{n-1}}}{\gamma+\alpha \cdot y_{n}+\beta \cdot y_{n-1}}, n=0,1, \cdots,
$$

where the parameters $\alpha, \beta$ and $\gamma$ and the initial conditions are arbitrary positive numbers. In Section 2, the local asymptotic stability of the equilibrium point of Equation (1.4) was investigated by using the Linearized Stability Theorem. A suitable Lyapunov function for the analysis of the global asymptotic stability behavior was used, like the idea in $[8,9]$. Furthermore, the characterization of the stability was examined that depends on the conditions of the coefficients (see [10]). In Section 3, the semi-cycle of positive solutions was analyzed. All this results will be shown theoretical and by simulations at the end of the paper.

\section{Local and Global Asymptotic Stability Analysis}

In this section, we discuss the local and global asymptotic stability of the unique positive equilibrium point of Equation (1.4) by using the theorems in [4,8-10].

The equilibrium points of Equation (1.4) are the solutions of the equation

$$
\bar{y}=\frac{(\alpha+\beta) \mathrm{e}^{-\bar{y}}}{\gamma+(\alpha+\beta) \bar{y}} .
$$

Set

$$
f(y)=\frac{(\alpha+\beta) \mathrm{e}^{-y}}{\gamma+(\alpha+\beta) y}-y
$$

for $y=0$ and $y \rightarrow \infty$ we obtain respectively, 


$$
f(0)=\frac{\alpha+\beta}{\gamma}>0, \lim _{y \rightarrow \infty} f(y)=-\infty
$$

and

$$
f^{\prime}(y)=\frac{-(\alpha+\beta) \mathrm{e}^{-y}[\gamma+(\alpha+\beta)(y+1)]}{[\gamma+(\alpha+\beta) y]^{2}}-1 .
$$

It follows that Equation (2.1) has exactly one solution $\bar{y}$. From this result Equation (1.4) has a unique equilibrium $\bar{y}$.

The linearized equation and the characteristic equation associated with Equation (1.4) about the equilibrium $\bar{y}$ is

$$
\begin{aligned}
& x_{n+1}+\frac{\alpha\left(\mathrm{e}^{-\bar{y}}+\bar{y}\right)}{\gamma+(\alpha+\beta) \bar{y}} x_{n}+\frac{\beta\left(\mathrm{e}^{-\bar{y}}+\bar{y}\right)}{\gamma+(\alpha+\beta) \bar{y}} x_{n-1}=0, \\
& n=0,1, \cdots
\end{aligned}
$$

and

$$
\lambda^{2}+\frac{\alpha\left(\mathrm{e}^{-\bar{y}}+\bar{y}\right)}{\gamma+(\alpha+\beta) \bar{y}} \lambda+\frac{\beta\left(\mathrm{e}^{-\bar{y}}+\bar{y}\right)}{\gamma+(\alpha+\beta) \bar{y}}=0,
$$

respectively.

Theorem 2.1. The following statements are true.

1) Every solution of Equation (1.4) is bounded if $0<y_{n}$.

2) The equilibrium point of Equation (1.4) is bounded if $0<\bar{y}$.

Proof.

1) Suppose that $0<y_{n}$. Let $\left\{y_{n}\right\}_{n=-1}^{\infty}$ be a solution of Equation (1.4). We have

$$
\begin{aligned}
& 0<y_{n+1}=\frac{\alpha \mathrm{e}^{-y_{n}}+\beta \mathrm{e}^{-y_{n-1}}}{\gamma+\alpha y_{n}+\beta y_{n-1}} \\
& <\frac{\alpha \mathrm{e}^{-y_{n}}+\beta \mathrm{e}^{-y_{n-1}}}{\gamma}<\frac{(\alpha+\beta) \mathrm{e}^{0}}{\gamma}=\frac{(\alpha+\beta)}{\gamma},
\end{aligned}
$$

which gives that every positive solution of the Equation (1.4) is bounded. Thus 1) is true.

2) Assume that $0<\bar{y}$. Then

$$
\begin{aligned}
0 & <\bar{y}=\frac{\alpha \mathrm{e}^{-\bar{y}}+\beta \mathrm{e}^{-\bar{y}}}{\gamma+\alpha \bar{y}+\beta \bar{y}}<\frac{\alpha \mathrm{e}^{-\bar{y}}+\beta \mathrm{e}^{-\bar{y}}}{\gamma} \\
& <\frac{(\alpha+\beta) \mathrm{e}^{0}}{\gamma}=\frac{(\alpha+\beta)}{\gamma},
\end{aligned}
$$

which implies that 2) is also true.

Theorem 2.2 Let $\alpha>\beta$. If

$$
(\alpha+\beta)<\gamma \mathrm{e}^{\frac{-\gamma+\sqrt{\gamma^{2}+4(\alpha+\beta) \gamma}}{2(\alpha+\beta)}},
$$

then the positive equilibrium point of Equation (1.4) is locally asymptotically stable.

Proof. From the Linearized Stability Theorem, we can write

$$
\left|-\frac{\alpha\left(\mathrm{e}^{-\bar{y}}+\bar{y}\right)}{\gamma+(\alpha+\beta) \bar{y}}\right|<1+\frac{\beta\left(\mathrm{e}^{-\bar{y}}+\bar{y}\right)}{\gamma+(\alpha+\beta) \bar{y}}<2 .
$$

The inequality (2.8) can be shown under two cases;

$$
\begin{aligned}
& \text { 1) }\left|-\frac{\alpha\left(\mathrm{e}^{-\bar{y}}+\bar{y}\right)}{\gamma+(\alpha+\beta) \bar{y}}\right|<1+\frac{\beta\left(\mathrm{e}^{-\bar{y}}+\bar{y}\right)}{\gamma+(\alpha+\beta) \bar{y}} \\
& \text { 2) } 1+\frac{\beta\left(\mathrm{e}^{-\bar{y}}+\bar{y}\right)}{\gamma+(\alpha+\beta) \bar{y}}<2 .
\end{aligned}
$$

From 2), we get

$$
\bar{y}>\frac{\beta \mathrm{e}^{-\bar{y}}-\gamma}{\alpha} .
$$

By 1), we will have

$$
0<(\alpha+\beta) \mathrm{e}^{-\bar{y}}+2(\alpha+\beta) \bar{y}+\gamma,
$$

which always holds and since $\alpha>\beta$, we can also obtain

$$
\bar{y}>\frac{(\alpha-\beta) \mathrm{e}^{-\bar{y}}-\gamma}{2 \beta} .
$$

Considering both (2.9) and (2.11), if

$$
\frac{(\alpha-\beta) \mathrm{e}^{-\bar{y}}-\gamma}{2 \beta}>\frac{\beta \mathrm{e}^{-\bar{y}}-\gamma}{\alpha},
$$

then we have

$$
(\alpha+\beta) \mathrm{e}^{-\bar{y}}<\gamma
$$

Rewriting (2.12), we get

$$
(\alpha+\beta) \bar{y}^{2}+\gamma \bar{y}-\gamma<0 .
$$

In view of (2.12) and (2.13), we obtain

$$
(\alpha+\beta)<\gamma \mathrm{e}^{\frac{-\gamma+\sqrt{\gamma^{2}+4(\alpha+\beta) \gamma}}{2(\alpha+\beta)}} .
$$

Theorem 2.3. Let the conditions in Theorem 2.2 hold and assume that $\bar{y}_{1}$ and $\bar{y}_{2}$ are the equilibrium points of Equation (1.4), which parameters have the conditions $\gamma_{2}<\gamma_{1}<\frac{(\alpha+\beta) \beta}{\alpha}$. If the parameter $\gamma$ decreases, then the local stability of the positive equilibrium point

$$
\bar{y}=\frac{-(\alpha \gamma-(\alpha+\beta) \beta)+\sqrt{(\alpha \gamma-(\alpha+\beta) \beta)^{2}+4 \alpha(\alpha+\beta)^{2}}}{2 \alpha(\alpha+\beta)}
$$


decreases also.

Proof. By the Linearized Stability Theorem, we have

$$
\left|-\frac{\alpha\left(\mathrm{e}^{-\bar{y}}+\bar{y}\right)}{\gamma+(\alpha+\beta) \bar{y}}\right|<1+\frac{\beta\left(\mathrm{e}^{-\bar{y}}+\bar{y}\right)}{\gamma+(\alpha+\beta) \bar{y}}<2 .
$$

Let

$$
\left|-\frac{\alpha\left(\mathrm{e}^{-\bar{y}}+\bar{y}\right)}{\gamma+(\alpha+\beta) \bar{y}}\right|=1 .
$$

From (2.16), computations will give us

$\alpha(\alpha+\beta) \bar{y}^{2}-((\alpha+\beta) \beta-\alpha \gamma) \bar{y}-(\alpha+\beta) \gamma=0$,

where we get the positive equilibrium point

$$
\bar{y}=\frac{(\alpha+\beta) \beta-\alpha \gamma+\sqrt{((\alpha+\beta) \beta-\alpha \gamma)^{2}+4 \alpha(\alpha+\beta)^{2}}}{2 \alpha(\alpha+\beta)} .
$$

Let us write

$$
\bar{y}_{1}=\frac{(\alpha+\beta) \beta-\alpha \gamma_{1}+\sqrt{\left((\alpha+\beta) \beta-\alpha \gamma_{1}\right)^{2}+4 \alpha(\alpha+\beta)^{2}}}{2 \alpha(\alpha+\beta)}
$$

and

$$
\bar{y}_{2}=\frac{(\alpha+\beta) \beta-\alpha \gamma_{2}+\sqrt{\left((\alpha+\beta) \beta-\alpha \gamma_{2}\right)^{2}+4 \alpha(\alpha+\beta)^{2}}}{2 \alpha(\alpha+\beta)},
$$

where $\gamma_{2}<\gamma_{1}<\frac{(\alpha+\beta) \beta}{\alpha}$.

Considering both (2.18) and (2.19), we get

$$
\bar{y}_{1}<\bar{y}_{2} \text {. }
$$

On the other side, from (2.15), we will investigate

$$
\frac{\beta\left(\mathrm{e}^{-\bar{y}}+\bar{y}\right)}{\gamma+(\alpha+\beta) \bar{y}}<1 .
$$

From (2.21) assume that

$$
\frac{\beta\left(\mathrm{e}^{-\bar{y}_{1}}+\bar{y}_{1}\right)}{\gamma+(\alpha+\beta) \bar{y}_{1}}<\frac{\beta\left(\mathrm{e}^{-\bar{y}_{2}}+\bar{y}_{2}\right)}{\gamma+(\alpha+\beta) \bar{y}_{2}}<1 .
$$

Considering the conditions in Theorem 2.2, if furthermore (2.22) holds, than the stability of $\bar{y}_{2}$ is weaker than $\bar{y}_{1}$. By computing (2.22), we obtain

$$
\begin{aligned}
& \gamma\left(\mathrm{e}^{-\bar{y}_{2}}-\mathrm{e}^{-\bar{y}_{1}}\right)+(\alpha+\beta)\left(\bar{y}_{1} \mathrm{e}^{-\bar{y}_{2}}-\bar{y}_{2} \mathrm{e}^{-\bar{y}_{1}}\right) \\
& +\gamma\left(\bar{y}_{2}-\bar{y}_{1}\right)>0 .
\end{aligned}
$$

This inequality can be also written in the form

$$
\begin{aligned}
& \gamma\left(\bar{y}_{2}^{2}-\bar{y}_{1}^{2}\right)+\frac{\gamma^{2}}{\alpha+\beta}\left(\bar{y}_{2}-\bar{y}_{1}\right)+\gamma\left(\bar{y}_{2}-\bar{y}_{1}\right) \\
& +(\alpha+\beta) \bar{y}_{1} \bar{y}_{2}\left(\bar{y}_{2}-\bar{y}_{1}\right)>0 .
\end{aligned}
$$

From (2.20) we get

$$
\gamma\left(\bar{y}_{2}+\bar{y}_{1}\right)+\frac{\gamma^{2}}{\alpha+\beta}+\gamma+(\alpha+\beta) \bar{y}_{1} \bar{y}_{2}>0,
$$

which always holds. This completes the proof.
Theorem 2.4. Suppose that $\left\{y_{n}\right\}_{-1}^{\infty}$ is a monoton decreasing solution of Equation (1.4) and assume that the conditions in Theorem 2.2 hold. If

$$
y_{n}>2 \bar{y}
$$

then the positive equilibrium point of Equation (1.4) is globally asymptotically stable.

Proof. We consider a Lyapunov function $V(n)$ defined by

$$
V(n)=\left\{y_{n}-\bar{y}\right\}^{2}, n=0,1,2, \cdots
$$

The change along the solutions of Equation (1.4) is

$$
\begin{aligned}
\Delta V(n) & =V(n+1)-V(n) \\
& =\left\{y_{n+1}-y_{n}\right\}\left\{y_{n+1}+y_{n}-2 \bar{y}\right\} .
\end{aligned}
$$

From (2.28) we can write

and

$$
y_{n+1}-y_{n}=\frac{\alpha \cdot \mathrm{e}^{-y_{n}}+\beta \cdot \mathrm{e}^{-y_{n-1}}}{\gamma+\alpha \cdot y_{n}+\beta \cdot y_{n-1}}-y_{n}
$$

$$
y_{n+1}+y_{n}-2 \bar{y}=\frac{\alpha \cdot \mathrm{e}^{-y_{n}}+\beta \cdot \mathrm{e}^{-y_{n-1}}}{\gamma+\alpha \cdot y_{n}+\beta \cdot y_{n-1}}+y_{n}-2 \bar{y} .
$$

It can be compute that by using the hypothesis we have $\Delta V(n)<0$, which is the condition for the global asymptotic stability of the positive equilibrium point of Equation (1.4).

\section{The Semi-Cycle and Oscillation}

In this section, we consider the semi-cycle and oscillation of the positive solutions of Equation (1.4). 


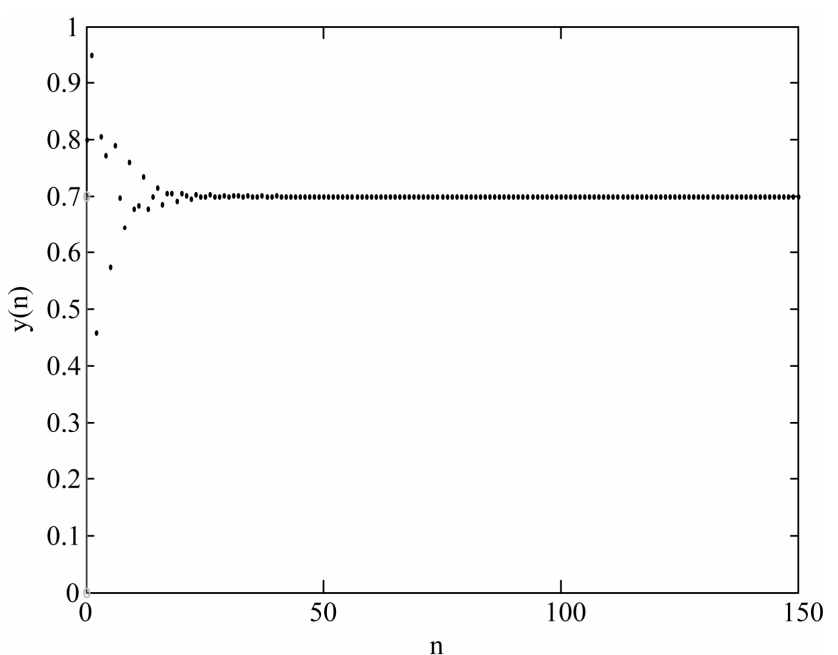

(a)

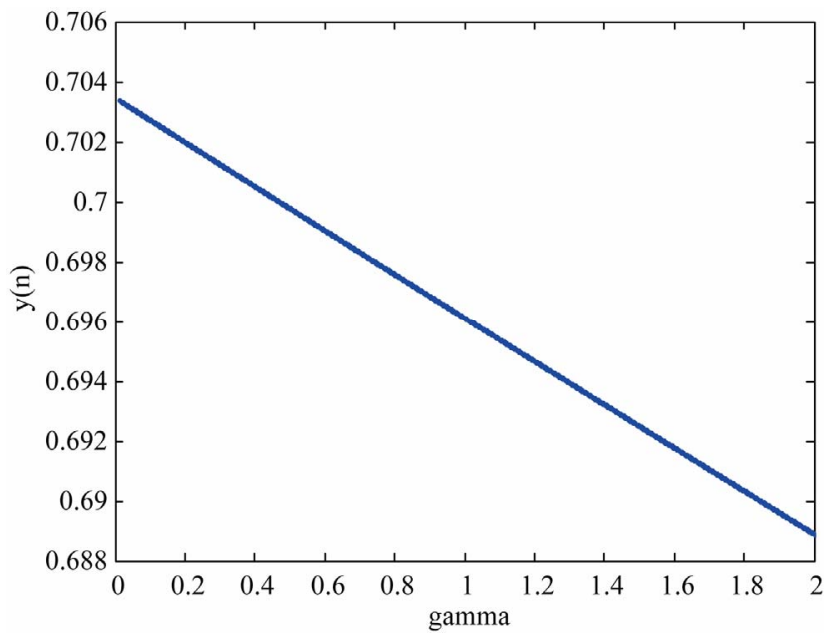

(c)

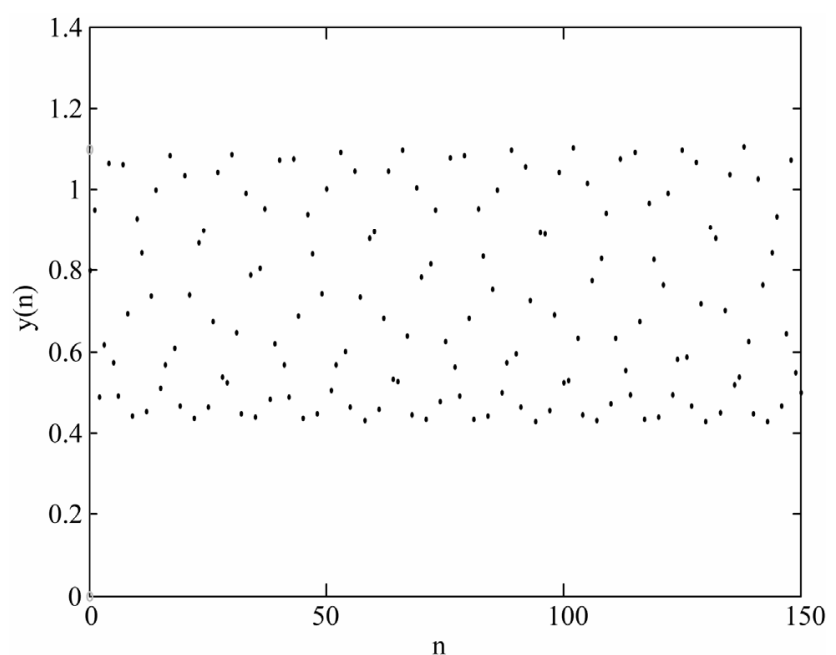

(b)

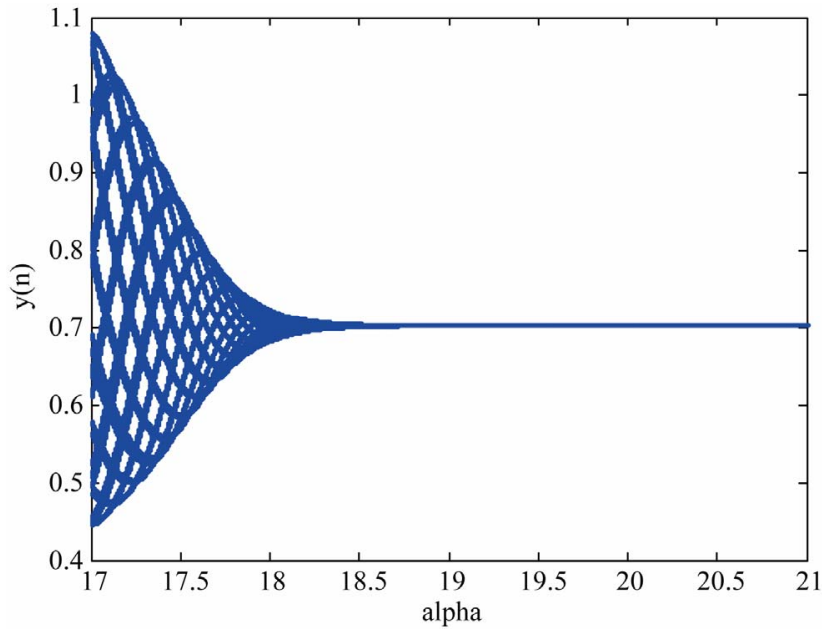

(d)

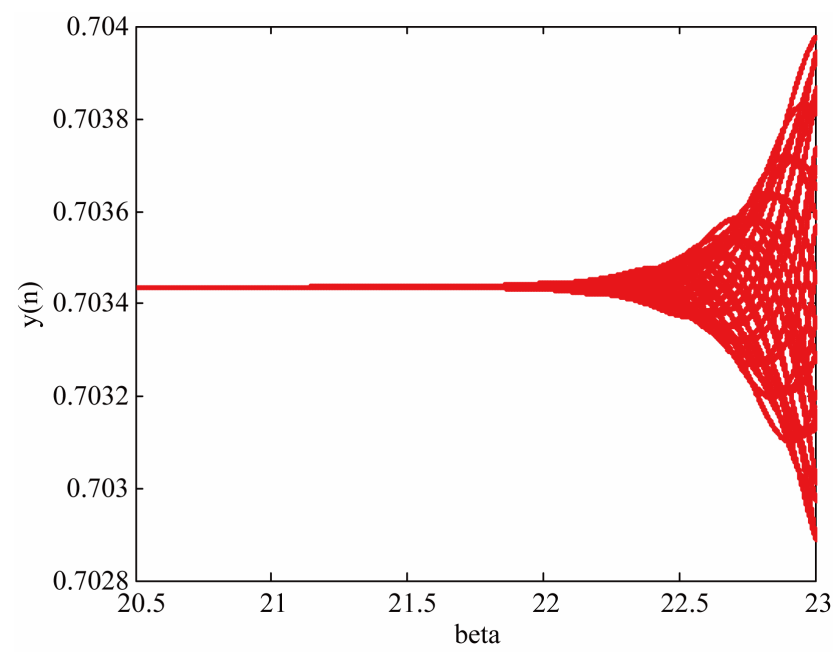

(e)

Figure 1. (a) Local stable behavior of Equation (1.4) for $\alpha=30, \beta=20, \gamma=0.3, y(-1)=0.8$ and $y(0)=0.95$; (b) Unstable behavior of Equation (1.4) for $\alpha=20, \beta=30, \gamma=0.3, y(-1)=0.8$ and $y(0)=0.9$; (c) Stability analysis for $\alpha=30, \beta=20, \gamma \in[0.01,2]$ $y(-1)=0.8$ and $y(0)=0.95$; (d) Diagram of the solutions of Equation (1.4) for $\beta=30, \alpha \in[17,21], \gamma \in 0.03, y(-1)=0.8$ and $y(0)$ $=0.95$; (e) Diagram of the solution of Equation (1.4) for $\alpha=17.2, \beta \in[20.5,23], \gamma \in 0.003, y(-1)=0.8$ and $y(0)=0.95$. 
By [8], a positive semi-cycle of a solution $\{x(n)\}_{n=-1}^{\infty}$ of $x(n+1)=f(x(n), x(n-1))$ consists of a "string" of terms $\{x(k), x(k+1), \cdots, x(m)\}$, all greater than or equal to the equilibrium $\bar{x}^{*}$, with $k \geq-1$ and $m \leq \infty$ and such that either $k=-1$ or $k>-1$ and $x(k-1)<\bar{x}$ and either $m=\infty$ or $m<\infty$ and $x(m+1)<\bar{x}$.

A negative semi-cycle of a solution $\{x(n)\}_{n=-1}^{\infty}$ of $x(n+1)=f(x(n), x(n-1))$ consists of a "string" of terms $\{x(k), x(k+1), \cdots, x(m)\}$, all less than the equilibrium $\bar{x}^{*}$, with $k \geq-1$ and $m \leq \infty$ and such that either $k=-1$ or $k>-1$ and $x(k-1) \geq \bar{x}$ and either $m=\infty$ or $m<\infty$ and $x(m+1) \geq \bar{x}$.

Theorem B ([9]) Assume that

$f \in C[(0, \infty) x(0, \infty),(0, \infty)]$ and that $f(x, y)$ is decreasing in both arguments. Let $\bar{y}$ be a positive equilibrium of $y(n+1)=f(y(n), y(n-1))$. Then every oscillatory solution of the difference equation

$y(n+1)=f(y(n), y(n-1))$ has semi-cycle of length at most two.

Theorem 3.2. Let $f(x, y)=\frac{\alpha \cdot \mathrm{e}^{-x}+\beta \cdot \mathrm{e}^{-y}}{\gamma+\alpha \cdot x+\beta \cdot y}$ be a function such that $f \in C[(0, \infty) x(0, \infty),(0, \infty)]$. Then every oscillatory solution of Equation (1.4) has semi-cycle of length at most two.

Proof. By the Theorem B, we can write Equation (1.4) such as

$$
f(x, y)=\frac{\alpha \cdot \mathrm{e}^{-x}+\beta \cdot \mathrm{e}^{-y}}{\gamma+\alpha \cdot x+\beta \cdot y} .
$$

The first derivative of (3.1) with respect to $x$ and $y$ are

$$
\frac{\partial f}{\partial x}=\frac{-\alpha \mathrm{e}^{-x}(\gamma+\alpha \cdot x+\beta \cdot y)-\alpha\left(\alpha \cdot \mathrm{e}^{-x}+\beta \cdot \mathrm{e}^{-y}\right)}{(\gamma+\alpha \cdot x+\beta \cdot y)^{2}}
$$

and

$$
\frac{\partial f}{\partial y}=\frac{-\beta \mathrm{e}^{-x}(\gamma+\alpha \cdot x+\beta \cdot y)-\beta\left(\alpha \cdot \mathrm{e}^{-x}+\beta \cdot \mathrm{e}^{-y}\right)}{(\gamma+\alpha \cdot x+\beta \cdot y)^{2}},
$$

respectively. These derivatives are less than zero if

$$
-\alpha \mathrm{e}^{-x}(\gamma+\alpha \cdot x+\beta \cdot y)-\alpha\left(\alpha \cdot \mathrm{e}^{-x}+\beta \cdot \mathrm{e}^{-y}\right)<0
$$

and

$$
-\beta \mathrm{e}^{-x}(\gamma+\alpha \cdot x+\beta \cdot y)-\beta\left(\alpha \cdot \mathrm{e}^{-x}+\beta \cdot \mathrm{e}^{-y}\right)<0
$$

respectively. Since the parameters are positive and the variables $\mathrm{x}$ and $\mathrm{y}$ are in a positive interval, (3.4) and (3.5) will be always hold. This completes the proof.

Example 1 In this Example, Figure 1(a) show the local stability of Equation (1.4) for the parameters $\alpha=30$, $\beta=20, \gamma=0.3, y(-1)=0.8$ and $y(0)=0.95$ by using the conditions in Theorem 2.2. The parameters $\alpha=20, \quad \beta=30 \quad \gamma=0.3$ and the initial conditions $y(-1)=0.8$ and $y(0)=0.95$ are selected to show in Figure 1(b) the unstable behavior of the solutions of Equation (1.4). In Figure 1(c), we can show that by decreasing of the parameter $\gamma$ the local stability get be weaker. At last, Figures 1(d) and (e) show the diagram of the solutions of Equation (1.4) for $\alpha \in[17,21]$, $\beta=25, \gamma=0.003, y(-1)=0.8$ and $y(0)=0.95$ and for $\alpha=17.2, \quad \gamma=0.003, \quad \beta \in[20.5,23], \quad y(-1)=0.8$ and $y(0)=0.95$, respectively. This give us the relation between the parameters $\alpha$ and $\beta$, which have an important role by the stability analysis of Equation (1.4), as shown in Theorem 2.2 and Theorem 2.4.

\section{REFERENCES}

[1] H. El-Metwally, E. A. Grove, G. Ladas, R. Levins and M. Radin, "On the Difference Equation $x_{n+1}=\alpha+\beta x_{n-1} \mathrm{e}^{-x_{n}}$," Nonlinear Analysis, Vol. 47, No. 7, 2001, pp. 4623-4634. doi:10.1016/S0362-546X(01)00575-2

[2] I. Ozturk, F. Bozkurt and S. Ozen, “On the Difference Equation $y_{n+1}=\frac{\alpha+\beta \mathrm{e}^{-y_{n}}}{\gamma+y_{n-1}}$," Applied Mathematics and Computations, Vol. 181, No. 2, 2006, pp. 1387-1393. doi:10.1016/j.amc.2006.03.007

[3] I. Ozturk, F. Bozkurt and S. Ozen, "Global Asymptotic Behavior of the Difference Equation

$y_{n+1}=\frac{\alpha \cdot \mathrm{e}^{-\left(n y_{n}+(n-k) y_{n-k}\right)}}{\beta+n y_{n}+(n-k) y_{n-k}}$," Applied Mathematic Letters, Vol. 22, No. 4, 2009, pp. 595-599. doi:10.1016/j.aml.2008.06.037

[4] C. H. Gibbons, M. R. S. Kulenovic, G. Ladas and H. D. Voulov, "On the Trichotomy Character of

$x_{n+1}=\left(\alpha+\beta x_{n}+\gamma x_{n-1}\right) /\left(A+x_{n}\right)$," Journal of Difference Equations and Applications, Vol. 8, No. 1, 2002, pp. 7592. doi:10.1080/10236190211940

[5] M. M. El-Afifi and A. M. Ahmed, "On the Difference Equation $\quad x_{n+1}=\frac{\alpha+\alpha x_{n}+\alpha x_{n-1}+\cdots+\alpha x_{n-k+2}}{x_{n-k+1}}$, Applied Mathematics and Computations, Vol. 144, No. 2-3, 2003, pp. 537-542. doi:10.1016/S0096-3003(02)00429-0

[6] W. S. He and W. T. Li, "Attractivity in a Nonlinear Delay Difference Equation,” Applied Mathematics E-Notes, Vol. 4, 2004, pp. 48-53.

[7] S. Ozen, I. Ozturk and F. Bozkurt, "On the Recursive Sequence $y_{n+1}=\frac{\alpha+y_{n-1}}{\beta+y_{n}}+\frac{y_{n-1}}{y_{n}}$," Applied Mathematics and Computation, Vol. 188, No. 1, 2007, pp. 180-188. doi:10.1016/j.amc.2006.09.106

[8] K. Cunningham, et al., "On the Recursive Sequence $x_{n+1}=\frac{\alpha+\beta x_{n}}{B x_{n}+C x_{n-1}}$,” Nonlinear Analysis, Vol. 47, No. 7, 2001, pp. 4603-4614. 
doi:10.1016/S0362-546X(01)00573-9

[9] C. Gibbons, M. R. S. Kulenovic and G. Ladas, "On the Recursive Sequence $x_{n+1}=\frac{\alpha+\beta x_{n-1}}{\gamma+x_{n}}$, Mathematical Sciences Research Hot-Line, Vol. 4, No. 2, 2000, pp. 1-
11.

[10] C. Celik, H. Merdan, O. Duman and O. Akın, "Allee Effects on Population Dynamics with Delay," Chaos, Solitons and Fractals, Vol. 37, No. 1, 2008, pp. 65-74. 